\title{
Minimal topological actions do not determine the measurable orbit equivalence class
}

\author{
Tullio Ceccherini-Silberstein and Gábor Elek
}

\begin{abstract}
We construct an amenable action $\Phi$ of a non-amenable group $\Gamma$ on a discrete space. This action extends to a minimal topological action $\widetilde{\Phi}$ of $\Gamma$ on a Cantor set $C$. We show that $\widetilde{\Phi}$ is non-uniquely ergodic and furthermore there exist ergodic invariant measures $\mu_{1}$ and $\mu_{2}$ such that $\left(\widetilde{\Phi}, C, \mu_{1}\right)$ and $\left(\widetilde{\Phi}, C, \mu_{2}\right)$ are not orbit equivalent measurable equivalence relations. This also provides an instance of the failure of equivalence between the notions of "global" and "local" amenability for countable equivalence relations.
\end{abstract}

Mathematics Subject Classification (2000). 37A20, 43A07, 28D15, 37A15.

Keywords. Minimal actions, measurable equivalence relations, orbit equivalence, amenable actions, amenable graphs, hyperfiniteness.

\section{Introduction}

J. Oxtoby [27] constructed an example of a minimal, non-uniquely ergodic $\mathbb{Z}$-action on a compact metric space (the action induced by the shift on a sequence space).

H. Dye [5] showed that any two (non-atomic) probability measure preserving ergodic $\mathbb{Z}$-actions are orbit equivalent.

Then D. Ornstein and B. Weiss [26] showed that any free ergodic probability measure preserving action of an amenable group is orbit equivalent to an ergodic $\mathbb{Z}$-action. In combination with Dye's result, this gives that all ergodic actions of amenable groups define the same equivalence relation, the hyperfinite relation.

In this paper we consider actions of non-amenable groups.

F. Greenleaf [12] posed the problem of the existence of amenable actions of nonamenable groups. E. K. van Douwen [4] provided the first examples. Recently, new examples were found by R. I. Grigorchuk and V.V. Nekrashevych [15] and by Y. Glasner and N. Monod [11].

In the present paper we construct a new example of amenable actions of nonamenable groups. More precisely we show the following. 
Example 1.1. There exists a countable, connected, amenable regular graph $G$ whose vertex set carries a faithful and transitive action $\Phi$ of the (non-amenable) group $\Gamma=\mathbb{Z}_{2} * \mathbb{Z}_{2} * \mathbb{Z}_{2} * \mathbb{Z}_{2}$.

This graph is a Schreier graph of $\Gamma$ and is properly 4-edge-colored. Moreover, it has dense holonomy, in the sense of Gromov [18] or, equivalently, in the language of the theory of Delone sets [22], it is repetitive. Also $G$ is generic (any two vertices have non-isomorphic spherical neighborhoods of sufficiently large radius).

With $G$ we then associate a compact metric space $X_{G}$, that we call the type space. In virtue of dense holonomy and genericity of $G$ we have that $X_{G}$ is homeomorphic to the Cantor set and that $G$ densely embeds into $X_{G}$. This way, the $\Gamma$-action $\Phi$ on $G$ naturally extends to a topological action $\widetilde{\Phi}$ on $X_{G}$. This action is faithful and minimal.

We then show that there exist two invariant measures $\mu_{1}$ and $\mu_{2}$ on $X_{G}$ such that the measurable equivalence relations $\left(\widetilde{\Phi}, X_{G}, \mu_{1}\right)$ and $\left(\widetilde{\Phi}, X_{G}, \mu_{2}\right)$ are not orbit equivalent. In particular, $\widetilde{\Phi}$ is not uniquely-ergodic. This is our main result, which we state as follows:

Theorem 1.2. There exists a minimal topological action $\tilde{\Phi}$ of a non-amenable group $\Gamma$ on a Cantor set $C$ for which there exist ergodic invariant measures $\mu_{1}$ and $\mu_{2}$ such that the measurable equivalence relation given by the systems $\left(\widetilde{\Phi}, C, \mu_{1}\right)$ and $\left(\widetilde{\Phi}, C, \mu_{2}\right)$ are not orbit equivalent. In fact one is hyperfinite, the other is non-hyperfinite.

To prove it, we use the theory of costs for countable measurable equivalence relations, initiated by G. Levitt [23] and developed by D. Gaboriau [8], [9], in combination with the Farrell-Varadarajan ergodic decomposition theorem.

Incidentally, our constriction also gives another instance of the failure of the equivalence between the "global" notion (hyperfiniteness) and the "local" notion (a.e. leaf is Følner) of amenability for countable equivalence relations. Indeed, by dense holonomy of $G$ and regularity of the action $\widetilde{\Phi}$, we have the following.

Theorem 1.3. Let $\widetilde{\Phi}$ be the minimal topological action of $\Gamma=\mathbb{Z}_{2} * \mathbb{Z}_{2} * \mathbb{Z}_{2} * \mathbb{Z}_{2}$ on $X_{G}$ (the natural extension of the action $\Phi$ of $\Gamma$ on the amenable graph $G$ ). Then all leaves are Folner.

Observe that whereas global amenability always implies local amenability, there exist (counter)examples of countable equivalence relations which are not hyperfinite but whose leaves (= orbits) are a.e. Følner: they were first given by V. A. Kaimanovich [19], [20]. 


\section{Amenable groups and amenable actions}

2.1. Amenable actions. Let $\Gamma$ be a countable group and $X$ a set (with no additional structure). A homomorphism $\Phi: \Gamma \rightarrow \operatorname{Sym}(X)$ from $\Gamma$ into the full permutation group of $X$ is called an action.

An action $\Phi: \Gamma \rightarrow \operatorname{Sym}(X)$ is amenable if there exists a $\Phi$-invariant finitelyadditive probability measure on $X$, that is, a map $\mu: \mathcal{P}(X) \rightarrow[0,1]$, where $\mathcal{P}(X)=$ $\{A \mid A \subseteq X\}$ is the set of all subsets of $X$ such that:

(i) $\mu(X)=1$ (normalization),

(ii) $\mu(A \cup B)=\mu(A)+\mu(B)-\mu(A \cap B)$; for all $A, B \subseteq X$ (finite additivity);

(iii) $\mu(\Phi(\gamma) A)=\mu(A)$, for all $A \subseteq X$ and $\gamma \in \Gamma$ ( $\Phi$-invariance).

A (discrete) group $\Gamma$ is amenable if the action $\lambda$ of $\Gamma$ on itself by left multiplication $\left(\lambda(\gamma) \gamma^{\prime}=\gamma \gamma^{\prime}\right.$, for all $\left.\gamma, \gamma^{\prime} \in \Gamma\right)$, is amenable.

2.2. Følner sequences and Schreier graphs. Let $G=G(V, E)$ be a graph with vertex set $V$ and edge set $E$. If $\{x, y\} \in E$ we also write $x \sim y$ and we say that the vertices $x$ and $y$ are neighbors. Let $x \in V$; if $\{x\} \in E$ we call this edge a loop at $x$. Note that we actually admit even multiple loops: if $x \in V$ has $k$ loops (or a loop with multiplicity $k$ ) we denote them by $(\{x\}, i) \in E$, for $i=1,2, \ldots, k$.

The boundary of a subset $F \subset V$, is defined by

$$
\partial F=\{x \in F \mid \text { there exists } y \in V \backslash F \text { such that } x \sim y\} .
$$

Suppose that $G$ is countable. One says that $G$ is Folner (or amenable) if it admits a Folner sequence, that is a sequence $\left\{F_{n}\right\}_{n \in \mathbb{N}}$ of non-empty finite subsets of $V$ such that $\lim _{n \rightarrow \infty} \frac{\left|\partial F_{n}\right|}{\left|F_{n}\right|}=0$. It can be shown that if $G$ is amenable, then there exist Følner sequences which are increasing $\left(F_{n} \subseteq F_{n+1}\right)$ and exhausting $\left(\bigcup_{n \in \mathbb{N}} F_{n}=V\right)$.

Let $\Gamma$ be a finitely generated group acting on a set $X$. Fix a finite and symmetric generating system $\Sigma$ and denote by $\mathcal{G}=\mathcal{E}(\Gamma, \Sigma, \Phi, X)$ the corresponding Schreier graph, that is, the graph with vertex set $V=X$ and $x \sim \Phi(\sigma) x$ for all $x \in X$ and $\sigma \in \Sigma$. If $X=\Gamma$ and $\Phi=\lambda$ one calls it the Cayley graph of $\Gamma$ with respect to $\Sigma$, simply denoted by $\mathcal{G}=\mathscr{H}(\Gamma, \Sigma)$.

Given a finitely generated group $\Gamma$ (with a symmetric generating system $\Sigma$ ) one has the following combinatorial characterization of amenability for group actions. The following conditions are equivalent:

(a) the action $\Phi: \Gamma \rightarrow \operatorname{Sym}(X)$ is amenable;

(b) the Schreier graph $\mathcal{E}(\Gamma, \Sigma, \Phi, X)$ is Følner.

In particular, with $\Phi=\lambda$, we have that the following are equivalent:

$\left(\mathrm{a}^{\prime}\right) \Gamma$ is amenable;

$\left(\mathrm{b}^{\prime}\right)$ the Cayley graph $\mathcal{G}(\Gamma, \Sigma)$ is Følner.

Note that if $\Gamma$ is amenable then all $\Gamma$-actions are amenable. 
2.3. Amenable actions of non-amenable groups. Greenleaf [12] raised the question on the existence of amenable actions of non-amenable groups, provided (to avoid trivialities) the actions are transitive and faithful.

This question was answered positively by van Douwen [4].

Recently, in [15] Grigorchuk and Nekrashevych provided two interesting new examples of amenable actions of non-amenable groups. In the first construction, $\Gamma$ is the free group $\mathbb{F}_{m}$ of rank $m \geq 2$ and $X$ is the homogeneous space $\mathbb{F}_{m} / H$ corresponding to a coamenable subgroup $H$ with trivial core $\left(\bigcap_{g \in \mathbb{F}_{m}} g^{-1} H g=\{1\}\right)$.

The second example is inspired by a "tree-wreathing" construction due to S. Sidki. Starting from an arbitrary non-amenable subgroup $\Gamma_{0}$ of $\operatorname{Aut}(\mathcal{T})$, the group of all automorphisms of an infinite spherically homogeneous rooted tree $\mathcal{T}$, they suitably extend it to a group $\Gamma$ acting on $\mathcal{T} \cup \partial \mathcal{T}$ (here $\partial \mathcal{T}$ denotes the boundary of $\mathcal{T}$ ). Then, the homogenous space is $X=\Gamma / P$ ) where $P$ is a parabolic subgroup, namely the stabilizer of a point in $\partial \mathcal{T}$.

We also refer to the recent paper by Glasner and Monod [11] who considered the class $\mathcal{A}$ consisting of all countable groups admitting a faithful and transitive amenable action.

2.4. Ergodic invariant measures and Oxtoby's example. Let $X$ be a compact metric space and $T: X \rightarrow X$ be a homeomorphism. A probability measure $\mu$ on $X$ is $T$-invariant if $\mu\left(T^{-1}(A)\right)=\mu(A)$ for all measurable subsets $A \subset X$ and ergodic it every $T$-invariant subset is $\mu$-null or $\mu$-conull: $A=T(A)$ implies $\mu(A) \in\{0,1\}$.

The transformation $T$ is minimal if each orbit is dense in $X$. Moreover, $T$ is uniquely ergodic if there exists a unique ergodic probability measure which is $T$-invariant.

In [27] Oxtoby constructed an example (a shift transformation on a sequence space) of a minimal, non-uniquely ergodic transformation on a compact metric space.

\section{From dense holonomy and genericity to minimal actions on the Cantor set}

3.1. Gromov's graphs of dense holonomy. Let $G=G(V, E)$ be a countable connected graph possibly with multiple loops. We also suppose that $G$ is regular of degree $K$ : for all $x \in V$ one has $|\{y \in V \mid y \sim x\}|=K$ (clearly, if $x$ has a loop with multiplicity $k$, then $K=|\{y \in V \mid y \neq x, y \sim x\}|+k)$.

A $K$-proper edge-coloring on $G$ is a map $\delta: E \rightarrow\{1,2, \ldots, K\}$ such that $\delta(e) \neq$ $\delta\left(e^{\prime}\right)$ for all distinct $e, e^{\prime} \in E$ with $e \cap e^{\prime} \neq \emptyset$; in words, adjacent edges (including multiple loops at a same vertex, if any) have different colors. If the graph $G$ has these properties we indicate it by $G=G(V, E, \delta, K)$.

Any such colorings $\delta$ define an action $\Phi=\Phi(\delta, K)$ of the group $\Gamma=\mathbb{Z}_{2} * \cdots * \mathbb{Z}_{2}$, the free product of $K$ copies of the group $\mathbb{Z}_{2}$ of order two, on the set $V$ of vertices 
of $G$. Indeed, letting $\Sigma$ denote the standard generating set for $\Gamma$, for any element $\gamma=$ $\sigma_{n} \sigma_{n-1} \ldots \sigma_{1}$ in $\Gamma\left(\sigma_{i} \in \Sigma\right)$ and $x \in V$, there exists a unique path $\left(x_{0}, x_{1}, \ldots, x_{n}\right)$ in $G$ such that:

- $x_{0}=x$;

- let $\left(x_{i}, y\right) \in E$ be the edge colored by $\sigma_{i}$; then $x_{i+1}=y$.

Then one sets $\Phi(\gamma)(x)=x_{n}$.

Let $r>0$. We call the spanned subgraph $B_{r}(x)=\left\{y \in V \mid d_{G}(x, y) \leq r\right\}$ of $G$ with the induced edge-coloring, the rooted colored $r$-ball around $x \in V$. Here $d_{G}$ denotes the geodesical distance on $G$.

Let $U_{G}^{r}$ be the set of rooted equivalence classes of these edge-colored rooted $r$ balls in $G$, that is, we consider two rooted $r$-balls $B_{r}(x)$ and $B_{r}(y)$ as equivalent if there exists a graph isomorphism $\theta: B_{r}(x) \rightarrow B_{r}(y)$ preserving the edge-coloring $\delta$ and mapping $x$ to $y$. Clearly $U_{G}^{r}$ is a finite set. For each $x \in V$ the $r$-type of $x$ is the element $\alpha_{r}(x) \in U_{G}^{r}$ representing the class of the $r$-ball around $x$.

Following Gromov [18], we call $G$ a graph of dense holonomy if, for any $\alpha \in U_{G}^{r}$, there exists an integer $m_{\alpha}$ such that any ball of radius $m_{\alpha}$ in $G$ contains at least one vertex $x$ with $r$-type $\alpha_{r}(x)=\alpha$. Note that in the theory of Delone sets such graphs are called repetitive [22].

Also, we say that the graph $G$ is generic if for any $x \neq y \in V$ there exists $r>0$ such that the $r$-types of $x$ and $y$ are different.

3.2. The associated topological action. Let $G=G(V, E, \delta, K)$ and $\Gamma$ be as in the previous section. We naturally associate with $G$ a compact metric space $X_{G}$ with a topological $\Gamma$-action by the construction below.

Let $r, s \in \mathbb{N}$ with $r<s$ and suppose that $\alpha \in U_{G}^{r}$ and $\beta \in U_{G}^{s}$. We write $\alpha \prec \beta$ if the $r$-ball around the root of $\beta$ is just $\alpha$, or equivalently, if $\alpha \supset \beta$.

Let $\tilde{\alpha}=\left\{\alpha_{1} \prec \alpha_{2} \prec \cdots\right\}$ be an infinite chain such that $\alpha_{r} \in U_{G}^{r}$ for all $r=1,2, \ldots$ These chains form the compact metric space $X_{G}$, the type space, where

$$
d_{X_{G}}\left(\left\{\alpha_{1} \prec \alpha_{2} \prec \cdots\right\},\left\{\beta_{1} \prec \beta_{2} \prec \cdots\right\}\right)=2^{-r}
$$

and $r$ is the smallest integer for which $\alpha_{r} \neq \beta_{r}$. We show below that the $\Gamma$-action on $G$ extends to $X_{G}$ in a natural way.

For each $x \in V$, we denote by $\pi(x)=\left\{\alpha_{1}(x) \prec \alpha_{2}(x) \prec \cdots\right\}$ the associated element in $X_{G}$, where $\alpha_{r}(x)$ is the $r$-type of $x$. Obviously, $G$ is generic if and only if $\pi: V \rightarrow X_{G}$ is an injective map.

Proposition 3.1. The action $\Phi$ extends to a topological action $\widetilde{\Phi}$ of $\Gamma$ on the metric space $X_{G}$.

Proof. First note that, by definition, the image of $\pi: V \rightarrow X_{G}$, where, for $x \in V$, $\pi(x)=\left\{\alpha_{1}(x) \prec \alpha_{2}(x) \prec \cdots\right\}$, is dense in $X_{G}$. Let $\tilde{\alpha}=\left\{\alpha_{1} \prec \alpha_{2} \prec \cdots\right\} \in X_{G}$ 
and consider an element $\gamma=\sigma_{n} \sigma_{n-1} \ldots \sigma_{1} \in \Gamma$, where $\sigma_{i} \in \Sigma$. For all $i=1,2, \ldots$ let $x_{i} \in V$ be such that $\alpha_{i+n}\left(x_{i}\right)=\alpha_{i+n}$. If $y_{i}=\Phi(\gamma)\left(x_{i}\right)$, setting $\beta_{i}=\alpha_{i}\left(y_{i}\right)$ one has $\beta_{i} \prec \beta_{i+1}$ and defines

$$
\widetilde{\Phi}(\gamma)(\tilde{\alpha}):=\left\{\beta_{1} \prec \beta_{2} \prec \cdots\right\} .
$$

Obviously $\widetilde{\Phi}(\gamma)$ is a continuous map and $\widetilde{\Phi}(\gamma)(\pi(x))=\pi(\Phi(\gamma)(x))$ for all $\gamma \in \Gamma$ and $x \in V$.

Proposition 3.2. If $\Phi$ is an amenable action of $\Gamma$, then $\widetilde{\Phi}$ admits an invariant measure on $X_{G}$.

Proof. First fix an ultrafilter $\omega$ on $\mathbb{N}$ and consider the associated ultralimit

$$
\lim _{\omega}: \ell^{\infty}(\mathbb{N}) \rightarrow \mathbb{R}
$$

Let $\left\{F_{n}\right\}_{n=1}^{\infty}$ be a Følner-sequence in $G$ with respect to $\Phi$. Let $\alpha \in U_{G}^{r}$ be an $r$-type of the Schreier graph. Denote by $\tau_{n}(\alpha)$ the number of vertices in the set $F_{n}$ which are of $r$-type $\alpha$. Then let

$$
\mu(\underline{\alpha}):=\lim _{\omega} \frac{\tau_{n}(\alpha)}{\left|F_{n}\right|},
$$

where $\underline{\alpha} \subset X_{G}$ is the clopen set of chains $\left\{\delta_{1} \prec \delta_{2} \prec \cdots\right\}$, with $\delta_{r}=\alpha$.

Now let $\left\{\beta_{r+1}^{1}, \beta_{r+1}^{2}, \ldots \beta_{r+1}^{k}\right\}$ be the set of $(r+1)$-types such that $\alpha \prec \beta_{r+1}^{i}$. Clearly,

$$
\tau_{n}(\alpha)=\sum_{i=1}^{k} \tau_{n}\left(\beta_{r+1}^{i}\right) .
$$

In other words, $\mu(\underline{\alpha})=\sum_{i=1}^{k} \mu\left(\beta_{r+1}^{i}\right)$, where $\underline{\beta_{r+1}^{i}} \subset X_{G}$ is the clopen set of chains with $(r+1)$-type $\beta_{r+1}^{i}$. This shows immediately that $\mu$ defines a Borel-measure on the compact metric space $X_{G}$. In order to prove that $\mu$ is $\widetilde{\Phi}$-invariant it is enough to show that

$$
\mu(\widetilde{\Phi}(\sigma)(\underline{\alpha}))=\mu(\underline{\alpha}),
$$

where $\sigma$ is one of the generators of $\Gamma$ (the involutions generating the free factors $\mathbb{Z}_{2}$ ). Let $\left\{\alpha_{1}, \alpha_{2}, \ldots, \alpha_{\ell}\right\} \subset U_{G}^{r+1}$ be the set of $(r+1)$-types in the Schreier graph of the action with the following property. The $r$-ball around $\Phi(\sigma)\left(x_{i}\right)$ is just $\alpha$, where $x_{i}$ is the root of $\alpha_{i}$. Observe that $\widetilde{\Phi}(\sigma)(\underline{\alpha})=\bigsqcup_{i=1}^{\ell} \underline{\alpha_{i}}$. Hence we need to prove that

$$
\mu(\underline{\alpha})=\sum_{i=1}^{\ell} \mu\left(\underline{\alpha_{i}}\right) .
$$


In order to check (3.1) it is enough to see that

$$
\lim _{n \rightarrow \infty} \frac{\left|\tau_{n}(\alpha)-\sum_{i=1}^{\ell} \tau_{n}\left(\alpha_{i}\right)\right|}{\left|F_{n}\right|}=0 .
$$

Let $F_{n}(\alpha)$ be the set of vertices in $F_{n}$ of $r$-type $\alpha$ and $F_{n}\left(\alpha_{i}\right)$ be the set of vertices in $F_{n}$ of $(r+1)$-type $\alpha_{i}$. Let us observe that:

- $F_{n}\left(\alpha_{i}\right) \cap F_{n}\left(\alpha_{j}\right)=\emptyset$ if $i \neq j$;

- for any $x \in F_{n}(\alpha)$, if $\Phi(\sigma)(x) \in F_{n}$, then $\Phi(\sigma)(x) \in F_{n}\left(\alpha_{i}\right)$ for some $i$;

- for any $x \in F_{n}\left(\alpha_{i}\right)$, if $\Phi(\sigma)(x) \in F_{n}$, then $\Phi(\sigma)(x) \in F_{n}(\alpha)$ (recall that $\sigma$ is an involution, namely $\sigma^{2}=1$ ).

Therefore,

$$
\left|\tau_{n}(\alpha)-\sum_{i=1}^{\ell} \tau_{n}\left(\alpha_{i}\right)\right| \leq 2\left|\partial F_{n}\right| .
$$

Thus (3.2) immediately follows from the Følner property.

3.3. Generic actions of dense holonomy. Let $G=G(V, E, \delta, K), \Gamma$ and $\Phi$ be as in Section3.1.

Suppose that $G=G(V, E, \delta, K)$ is generic resp. of dense holonomy. Then we say that the associated $\Gamma$-action $\Phi$ is generic resp. of dense holonomy.

Proposition 3.3. Suppose that $\Phi$ is of dense holonomy and generic. Then $X_{G}$ is homeomorphic to a Cantor set and the associated topological $\Gamma$ action $\widetilde{\Phi}$ is minimal.

Proof. Let $\alpha \in U_{G}^{r}$, then there exists $\beta \neq \beta^{\prime} \in U_{G}^{s}$ (for some $s>r$ ) such that $\alpha \prec \beta$ and $\alpha \prec \beta^{\prime}$, that is, the $r$-balls around the roots of $\beta$ and $\beta^{\prime}$ are of type $\alpha$. Indeed, let $x \in V$ a vertex of $r$-type $\alpha$. Then, by the dense holonomy property, there exists $y \in V$ of the same $r$-type. By genericity there exists some $s>r$ such that the $s$-types of $x$ and $y$ are different. Consequently, $X_{G}$ is a compact metric space with no isolated points, and thus, also in view of its ultrametric structure, it is homeomorphic to the Cantor set.

Now we prove minimality. Let $\tilde{\alpha}=\left\{\alpha_{1} \prec \alpha_{2} \prec \cdots\right\}$ and $\tilde{\beta}=\left\{\beta_{1} \prec \beta_{2} \prec \cdots\right\}$ be elements of $X_{G}$. It is enough to show that for any $r>0$ there exists $\gamma \in \Gamma$ such that $\widetilde{\Phi}(\gamma)(\tilde{\alpha})=\left\{\beta_{1} \prec \beta_{2} \prec \cdots \prec \beta_{r} \prec \beta_{r+1}^{\prime} \cdots\right\}$, that is, $d(\tilde{\beta}, \widetilde{\Phi}(\gamma)(\tilde{\alpha})) \leq 2^{-r}$. Let $m>r$ be an integer such that, by dense holonomy, any $m$-ball in the graph $G$ contains a vertex of $r$-type $\beta_{r}$. Consider a vertex $p \in V$ such that its $2 m$-type is $\alpha_{2 m}$. Let $q \in B_{m}(p)$ be a vertex of $r$-type $\beta_{r}$. Then there exists a group element $\gamma=\sigma_{\ell} \sigma_{\ell-1} \ldots \sigma_{1}, \underset{\sim}{ } \leq m$, such that $\Phi(\gamma)(p)=q$. By the definition of the induced topological action $\widetilde{\Phi}$ one has $\widetilde{\Phi}(\gamma)(\tilde{\alpha})=\left\{\beta_{1} \prec \beta_{2} \prec \cdots \prec \beta_{r} \prec \cdots\right\}$. 
Note. If the action of a group is generic but not of dense holonomy, the associated action on the type space may fail to be minimal. Consider, for instance, the group $D_{\infty}=\mathbb{Z}_{2} * \mathbb{Z}_{2}$, the infinite dihedral group generated by the symbols $A$ and $B$. We define an action on the positive integers $\mathbb{N}$ in the following way:

- $\Phi(A)(2 n-1)=2 n, n \geq 1$

- $\Phi(A)(2 n)=2 n-1, n \geq 1$;

- $\Phi(B)(2 n-1)=2 n-2, n>1$;

- $\Phi(B)(2 n)=2 n+1, n \geq 1$;

- $\Phi(B)(1)=1$.

It is easy to verify that $\Phi$ is generic. The associated compact metric space is $\{1,2,3, \ldots\} \cup\{\infty\}$, where

$$
\widetilde{\Phi}(A)(\infty)=\infty \quad \text { and } \quad \widetilde{\Phi}(B)(\infty)=\infty .
$$

Thus the action $\widetilde{\Phi}$ is not minimal.

\section{The main construction}

4.1. Technicalities. For our main construction we need a couple of simple lemmas of a purely graph theoretical flavour.

Lemma 4.1. Let $G(V, E)$ be a finite connected graph and $d$ be an integer larger than 2. Suppose that $V^{\prime} \subset V$ is a $2 d$-net, that is, if $x \neq y \in V^{\prime}$ then $d_{G}(x, y) \geq 2 d$. Also suppose that $\operatorname{diam} G \geq 2 d$. Then $\frac{\left|V^{\prime}\right|}{|V|} \leq \frac{1}{d}$.

Proof. By the diameter condition, for each $x \in V^{\prime}$ and $i=1,2, \ldots, d$ there exists $z_{x, i} \in V$ such that $d_{G}\left(x, z_{x, i}\right)=i$. Hence $\left|B_{d}(x)\right| \geq d$. Since the $d$-balls around the elements of a $2 d$-net are disjoint, the lemma follows.

Lemma 4.2. Let $s_{1}<s_{2}<\cdots<s_{n}$ be integers, $s_{i} \geq 10^{i+1}$. Suppose that $\operatorname{diam}(G)>10 s_{n}$. Then there exists a partition

$$
V=R_{1} \amalg R_{2} \amalg \cdots \amalg R_{n} \amalg Q
$$

such that

- $R_{i}$ is a $2 s_{i}$-net for all $i=1,2, \ldots, n$;

- for all $x \in V$ and $1 \leq i \leq n$ there exists $p_{i} \in R_{i}$ such that $d_{G}\left(p_{i}, x\right) \leq 10 s_{i}$. 
Proof. Let $R_{1} \subset V$ be a maximal $2 s_{1}$-net in $V$. By the previous lemma

$$
\frac{\left|R_{1}\right|}{|V|} \leq \frac{1}{s_{1}}
$$

Moreover, for the same reason, for any spanned subgraph $T \subseteq G$ with $\operatorname{diam}(T) \geq$ $2 s_{1}$ :

$$
\frac{\left|T \cap R_{1}\right|}{|T|} \leq \frac{1}{s_{1}}
$$

By maximality, for any $y \in V$ there exists $p_{1} \in R_{1}$ such that $d\left(p_{1}, y\right) \leq 2 s_{1} \leq 10 s_{1}$. Now let $R_{2} \subset V$ be a $2 s_{2}$-net which is maximal with respect to the property that $R_{1} \cap R_{2}=\emptyset$. Again, for any spanned subgraph $T \subseteq G$ with $\operatorname{diam}(T) \geq 2 s_{2}$ :

$$
\frac{\left|T \cap R_{1}\right|}{|T|} \leq \frac{1}{s_{1}}, \quad \frac{\left|T \cap R_{2}\right|}{|T|} \leq \frac{1}{s_{2}}
$$

Let $x$ be an arbitrary vertex of $V$. We need to prove that $B_{10 s_{2}}(x) \cap R_{2}$ is non-empty. If $B_{10 s_{2}}(x) \cap R_{2}=\emptyset$ then by maximality, $B_{5 s_{2}}(x)$ is completely filled with vertices from the set $R_{1}$. However, diam $B_{5 s_{2}}(x) \geq 2 s_{2}$, which is in contradiction with (4.1). Thus $p_{2}$ can be chosen as any element in $B_{10 s_{2}}(x) \cap R_{2}$. By induction, suppose that for $i=1,2, \ldots$ we have determined a $2 s_{i}$-net $R_{i} \subset V$ which is maximal with respect to the property that $R_{i} \cap R_{j}=\emptyset$ whenever $j<i$. Then, for any spanned subgraph $T \subseteq G$ with $\operatorname{diam}(T) \geq 2 s_{i}$ :

$$
\frac{\left|T \cap R_{1}\right|}{|T|} \leq \frac{1}{s_{1}}, \quad \frac{\left|T \cap R_{2}\right|}{|T|} \leq \frac{1}{s_{2}}, \quad \ldots, \quad \frac{\left|T \cap R_{i}\right|}{|T|} \leq \frac{1}{s_{i}} .
$$

Thus, when constructing the $2 s_{i+1}$-net $R_{i+1} \subset V$, in order to check the existence of $p_{i+1} \in B_{10 s_{i+1}}(x) \cap R_{i+1}$ for any $x \in V$, the same arguments for the case $i=1$ apply and $B_{10 s_{i+1}}(x) \cap R_{i+1}=\emptyset$ would contradict (4.3), as $\sum_{j=1}^{i} \frac{1}{s_{j}}<1$.

4.2. The construction. The goal of this section is to construct a

- faithful, transitive and amenable,

- generic,

- dense holonomy

$\Gamma$-action on a countable set $X$.

The countable set $X$ will be the vertex set of a graph $G=G(V, E, \delta, 4)$ (thus regular of degree 4 with a 4-proper edge-coloring) which is generic and of dense holonomy.

From now on we shall denote by $\Gamma$ the group $\mathbb{Z}_{2} * \mathbb{Z}_{2} * \mathbb{Z}_{2} * \mathbb{Z}_{2}$, freely generated by the elements $A, B, C, D$ of order two, and by $\widetilde{\Gamma}$ the subgroup generated by $A, B$ and $C$. 
We also fix a parameter $m>10$ to be specified later. We need two auxiliary graph sequences. The first one is $\left\{C_{i}\right\}_{i=1}^{\infty}$, where $C_{i}$ is the cycle of length $2 i$ edge-colored by $A$ and $B$. The second graph sequence is given in the following the way. The group $\widetilde{\Gamma}$ is residually finite (free product of finite groups), hence one can pick a decreasing sequence of finite index normal subgroups with trivial intersection:

$$
\widetilde{\Gamma} \triangleright N_{1} \triangleright N_{2} \triangleright N_{3} \triangleright \cdots, \quad \bigcap_{i=1}^{\infty} N_{i}=\{1\} .
$$

Then the 3-colored graph $K_{i}$ is the Cayley-graph of the finite group $\widetilde{\Gamma} / N_{i}$. Note that $\left\{K_{i}\right\}_{i=1}^{\infty}$ is a large girth sequence, that is, for any $s>0$, if $i$ is large enough, then $K_{i}$ does not contain cycles of length less than $s$.

Finally, we lexicographically enumerate the non-trivial elements in $\Gamma: A, B$, $C, D, A B, A C, A D, B A, \ldots$. Denote the corresponding $n$-th element by $\gamma_{n}=$ $\sigma_{k_{n}}^{(n)} \sigma_{k_{n}-1}^{(n)} \cdots \sigma_{2}^{(n)} \sigma_{1}^{(n)}$, where $\sigma_{i}^{(n)} \in \Sigma=\{A, B, C, D\}$; note that the word-length $k_{n}$ of $\gamma_{n}$ is at most $n$.

Step (1): Let $G_{0}\left(V_{0}, E_{0}\right)$ be the graph consisting of one single vertex $p$. Let $G_{1}\left(V_{1}, E_{1}\right)$ be the graph constructed in the following way. $H_{1}$ is a triangle, edgecolored by $A, B$ and $C$. The vertex $p$ is connected to a vertex $q$ of $H_{1}$ by an edge colored by $D$. Finally a vertex of $H_{1}$ which is not $q$ shall be called $r_{1}$. Thus we have graphs $G_{0} \subset G_{1}$. In the $n$-th step we shall have graphs $G_{0} \subset G_{1} \subset G_{2} \subset \cdots \subset G_{n}$. Finally let us set $s_{1}:=2 m, s_{2}=m^{2} 2^{\left|V\left(G_{1}\right)\right|}$.

Step (2): Let $H_{2}$ be a graph $C_{i}$, in such a way that diam $C_{i}>10 s_{2}$. By Lemma4.2 there exists a partition

$$
V\left(H_{2}\right)=R_{1}^{2} \coprod R_{2}^{2} \coprod Q^{2}
$$

where $R_{1}^{2}$ is a $2 s_{1}$-net, $R_{2}^{2}$ is a $2 s_{2}$-net satisfying the properties described in the lemma. Connect each point of $R_{1}^{2}$ to a copy of $G_{0}$ (a single vertex) with an edge colored by $D$. For one single vertex of $R_{2}^{2}$ connect a copy of $G_{1}$ (at the vertex $r_{1}$ !) with an edge colored by $D$. Let $x_{2}$ be a vertex of $Q^{2}$. Consider the first non-trivial element in $\Gamma$, namely $\gamma_{1}=A$. Let $y_{0}^{2}, y_{1}^{2}$ be two new vertices (i.e. distinct from any other vertex involved in the construction till now) and connect $x_{2}$ to $y_{0}^{2}$ by an edge colored by $D$ and connect $y_{0}^{2}$ to $y_{1}^{2}$ by an edge colored by $A$. Finally pick an other vertex of $Q^{2}$ and call it $r_{2}$.

Thus we constructed 4-colored graphs $G_{0} \subset G_{1} \subset G_{2}$. In $G_{2}$ we have a spanned subgraph $\mathrm{H}_{2}$ such that

$$
\frac{\left|V\left(G_{2}\right) \backslash V\left(H_{2}\right)\right|}{\left|V\left(G_{2}\right)\right|}<\frac{1}{m} .
$$


Indeed, $\left|V\left(G_{2}\right)\right|=\left(1+\left|V\left(G_{0}\right)\right|\right)\left|R_{1}^{2}\right|+\left(\left|V\left(G_{1}\right)\right|+\left|R_{2}^{2}\right|\right)+\left|Q^{2}\right|+2$ (the last term corresponds to $\left.\left|\left\{y_{0}^{2}, y_{1}^{2}\right\}\right|\right)$ and

$$
\begin{aligned}
\frac{\left|V\left(G_{2}\right) \backslash V\left(H_{2}\right)\right|}{\left|V\left(G_{2}\right)\right|} & =\frac{\left|R_{1}^{2}\right|+\left|V\left(G_{1}\right)\right|+2}{\left|V\left(G_{2}\right)\right|} \\
& \leq \frac{\left|R_{1}^{2}\right|+7}{\left|V\left(H_{2}\right)\right|} \leq \frac{1}{2 m}+\frac{7}{10 m^{2} 2^{4}}<1 / m .
\end{aligned}
$$

Set $s_{3}:=m^{3} 2^{\left|V\left(G_{2}\right)\right|}$.

Step (n): Suppose that we have already constructed the graphs $G_{0} \subset G_{1} \subset G_{2} \subset$ $\cdots \subset G_{n-1}$ with the following properties:

- if $i>1$, then $G_{i}$ contains a spanned subgraph $H_{i}$ such that

$$
\frac{\left|V\left(G_{i}\right) \backslash V\left(H_{i}\right)\right|}{\left|V\left(G_{i}\right)\right|}<\frac{1}{m}
$$

- $s_{i}=m^{i} 2^{\left|V\left(G_{i-1}\right)\right|}$

- $\left|V\left(H_{i-1}\right)\right|<\left|V\left(H_{i}\right)\right|$;

- for each $H_{i}$, we fixed a vertex $r_{i}$.

Let then $H_{n}$ be isomorphic to $C_{i}$ for $n$ even and to $K_{i}$ for $n$ odd, for some $i \in \mathbb{N}$ in such a way that $\operatorname{diam}\left(H_{n}\right) \geq 10 s_{n}$.

We apply Lemma 4.2 again:

$$
V\left(H_{n}\right)=R_{1}^{n} \amalg R_{2}^{n} \bigsqcup \cdots \amalg Q^{n},
$$

where $R_{i}^{n}$ is a $2 s_{i}$-net and for each $x \in V\left(H_{n}\right)$ there exists $p \in R_{i}^{n}$ such that

$$
d_{H_{n}}(p, x)<10 s_{i}
$$

For $i=1,2, \ldots, n-1$ we connect a copy of $G_{i-1}$ (at vertex $r_{i}$ ) to each vertex of $R_{i}^{n}$ by an edge colored by $D$. Then we connect one single copy of $G_{n-1}$ to $H_{n}$ by an edge colored by $D$ between $r_{n-1}$ and an arbitrary vertex in $R_{n}^{n}$. The remaining set $Q^{n}$ plays a different role: we just need two points in it, the first one $r_{n} \in Q^{n}$ to connect, at the following (inductive) steps, $G_{n}$ with the $H_{m}, m \geq n$, the second one, $x_{n} \in Q^{n} \backslash\left\{r_{n}\right\}$ to attach a path corresponding to the $n$-th word $\gamma_{n} \in \Gamma$ (which will guarantee the faithfulness of the $\Gamma$ action on $G$ ). More precisely, consider the $n$th element $\gamma_{n}=\sigma_{k_{n}}^{(n)} \sigma_{k_{n}-1}^{(n)} \ldots \sigma_{1}^{(n)}$.

- If $\sigma_{1}^{(n)}=D$, then choose new vertices $y_{1}^{n}, y_{2}^{n}, \ldots, y_{k_{n}}^{n}$ distinct from any previous vertices and connect the path $\left(y_{1}^{n}, y_{2}^{n}, \ldots, y_{k_{n}}^{n}\right)$ to $x_{n}$ by the edge $\left(x_{n}, y_{1}^{n}\right)$ colored by $D$ and let color the edges $\left(y_{i}^{n}, y_{i+1}^{n}\right)$ by $\sigma_{i+1}^{n}$. 
- If $\sigma_{1}^{(n)} \neq D$, then choose new vertices $y_{0}^{n}, y_{1}^{n}, y_{2}^{n}, \ldots, y_{k_{n}}^{n}$ distinct from any previous vertices and connect the path $\left(y_{0}^{n}, y_{1}^{n}, \ldots, y_{k_{n}}^{n}\right)$ to $x_{n}$ by the edge $\left(x_{n}, y_{1}^{n}\right)$ colored by $D$ and let color the edges $\left(y_{i}^{n}, y_{i+1}^{n}\right)$ by $\sigma_{i+1}^{n}$.

Note that we also have

$$
\frac{\left|V\left(G_{n}\right) \backslash V\left(H_{n}\right)\right|}{\left|V\left(G_{n}\right)\right|}<\frac{1}{m} .
$$

Indeed, arguing as in Step (2), we have

$$
\begin{aligned}
\left|V\left(G_{n}\right)\right|= & \left(1+\left|V\left(G_{0}\right)\right|\right)\left|R_{1}^{n}\right|+\left(1+\left|V\left(G_{1}\right)\right|\right)\left|R_{2}^{n}\right|+\cdots \\
& \cdots+\left(1+\left|V\left(G_{n-2}\right)\right|\right)\left|R_{n-1}^{n}\right|+\left(\left|R_{n}^{n}\right|+\left|V\left(G_{n-1}\right)\right|\right)+\left(\left|Q^{n}\right|+k_{n}\right),
\end{aligned}
$$

where $k_{n}$ corresponds to $\left|\left\{y_{1}^{n}, \ldots, y_{k_{n}}^{n}\right\}\right|$. Thus,

$$
\begin{aligned}
\frac{\left|V\left(G_{n}\right) \backslash V\left(H_{n}\right)\right|}{\left|V\left(G_{n}\right)\right|}= & \frac{1}{\left|V\left(G_{n}\right)\right|}\left[\left|V\left(G_{0}\right)\right|\left|R_{1}^{n}\right|+\left|V\left(G_{1}\right)\right|\left|R_{2}^{n}\right|+\cdots\right. \\
& \left.\cdots+\left|V\left(G_{n-2}\right)\right|\left|R_{n-1}^{n}\right|+\left|V\left(G_{n-1}\right)\right|+k_{n}\right] \\
\leq & \frac{1}{\left|V\left(H_{n}\right)\right|}\left[\left|V\left(G_{0}\right)\right|\left|R_{1}^{n}\right|+\left|V\left(G_{1}\right)\right|\left|R_{2}^{n}\right|+\cdots\right. \\
& \left.\cdots+\left|V\left(G_{n-2}\right)\right|\left|R_{n-1}^{n}\right|+\left|V\left(G_{n-1}\right)\right|+n\right] \\
\leq & \sum_{i=1}^{n-1}\left|V\left(G_{i-1}\right)\right| \frac{\left|R_{i}^{n}\right|}{\left|V\left(H_{n}\right)\right|}+\frac{\left|V\left(G_{n-1}\right)\right|}{\left|V\left(H_{n}\right)\right|}+\frac{n}{\left|V\left(G_{n}\right)\right|} \\
\leq & \sum_{i=1}^{n-1} \frac{\left|V\left(G_{i-1}\right)\right|}{m^{i} 2^{\left|V\left(G_{i-1}\right)\right|}}+\frac{\left|V\left(G_{n-1}\right)\right|}{\left|V\left(H_{n}\right)\right|}+\frac{n}{\left|V\left(G_{n}\right)\right|} \\
\leq & 1 / m .
\end{aligned}
$$

Note that our choice of the $s_{i}$ 's, namely $s_{i} \geq 10^{i+1}$, besides giving the obvious estimate $\sum_{i=1}^{\infty} \frac{1}{s_{i}}<1$ (which is needed, for instance, at the end of the proof of Lemma 4.2) is motivated by the fact that, later on, when considering Følner sequences in $G$, we want to have the "new" part $G_{n} \backslash G_{n-1}$ much larger than the "old" part $G_{n-1}$, so that the latter can be basically neglected in our computations.

Thus we constructed a sequence of graphs $G_{0} \subset G_{1} \subset \cdots \subset G_{n}$ and we proceed by induction. Set $G^{\prime}=\cup_{n=1}^{\infty} G_{n}$. For each $x \in V\left(G^{\prime}\right)$ of degree $\delta(x)<4$ we add $4-\delta(x)$ loops at $x$ and we label them with suitable different letters in $\{A, B, C, D\}$ in such a way that all edges incident to $x$ bear distinct labels. Denote the resulting 4-regular, properly 4-edge colored graph by $G$. In the next section, we prove that $G$ is generic, has dense holonomy and carries a faithful, transitive, amenable action of the group $\Gamma$. 
In Figure 1 we try to give an idea of how the chain of finite graphs $G_{0} \subset G_{1} \subset$ $G_{2} \subset \cdots \subset G_{n} \subset G_{n+1} \subset \cdots$ and the whole graph $G$ look like. It is purely illustrative and not completely realistic. For instance, we omitted the loops (so that the resulting graph is not 4-regular), and the shapes (for $n$ odd) and the sizes (e.g. $\left.\operatorname{diam}\left(H_{i}\right) \nsucceq 10 s_{i}\right)$ of the subgraphs $H_{n}$ do not completely satisfy the requirements from our definitions.

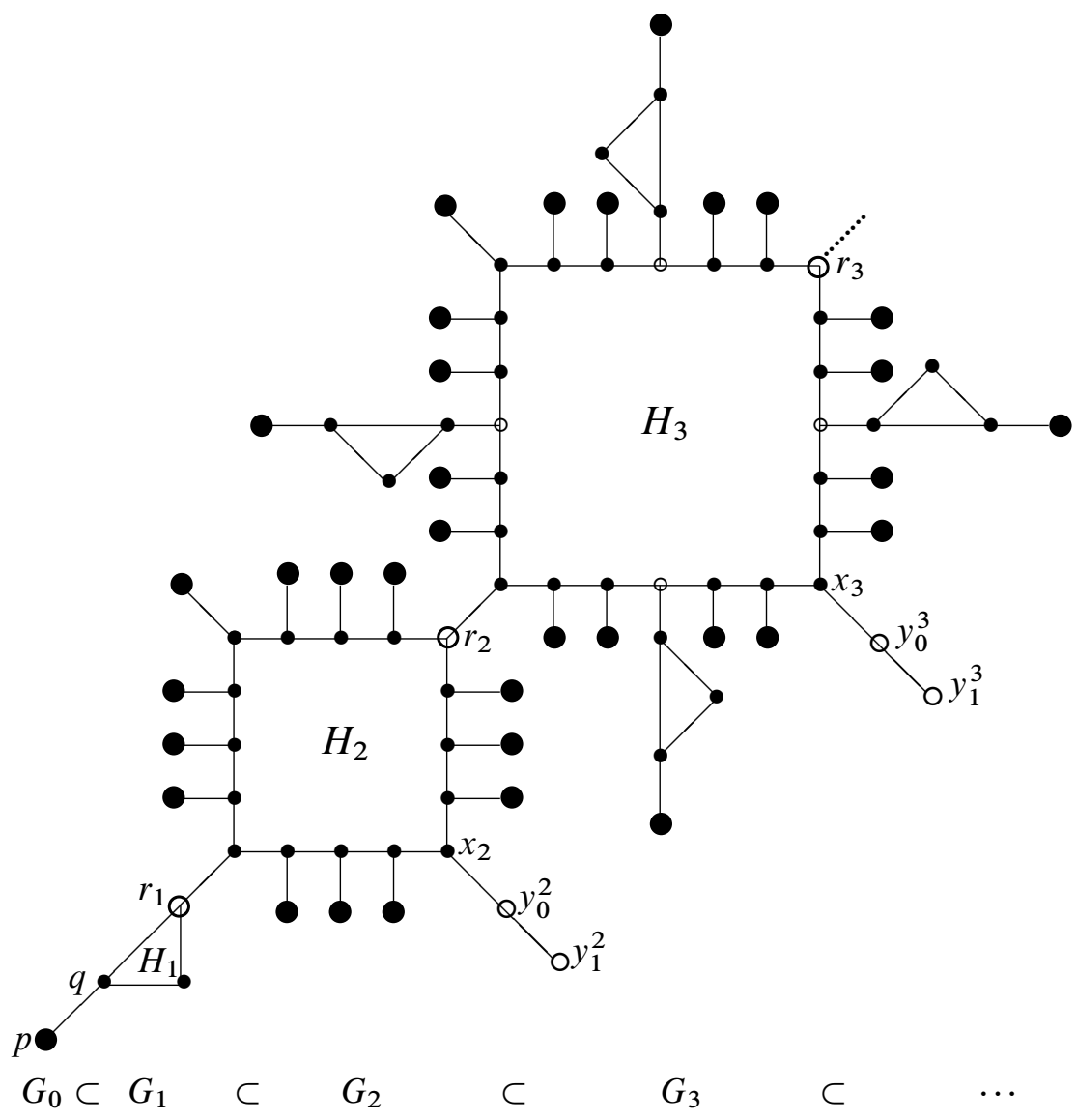

Figure 1. An illustration of the chain $G_{0} \subset G_{1} \subset G_{2} \subset \cdots$.

4.3. The graph $G$. In this section we check that the graph $G$ constructed in the previous section has indeed all the required properties.

Proposition 4.3. The action of $\Gamma$ is faithful, transitive and amenable. 
Proof. Transitivity is obvious from the connectivity of $G$. Faithfulness was ensured by the attachment of paths $\left(y_{1}^{n}, y_{2}^{n}, \ldots, y_{k_{n}}^{n}\right)$. The subgraphs $\left\{G_{n}\right\}_{n=1}^{\infty}$ are forming an increasing and exhausting Følner sequence, since $\partial G_{n}=\left\{r_{n}\right\}$.

Lemma 4.4. The single vertex $p$ of $G_{0}$ has the property that for any $q \in V(G)$, $q \neq p$, there exists $r$ such that $B_{r}(p)$ and $B_{r}(q)$ are not isomorphic.

Proof. Let $q \in V(G)$ be a vertex with three loops (otherwise the statement trivially holds). If $q$ was defined in Step (k), then there exists a spanned connected subgraph of $G$ which is isomorphic to $H_{k}$ such that the path from $q$ to the subgraph does not contain any vertex of a subgraph isomorphic to $H_{k-1}$. Indeed, $H_{k}$ itself can be chosen as the spanned subgraph. On the other hand, any path connecting $p$ to a spanned subgraph isomorphic to $H_{k}$ passes through such a vertex. In particular, $r=\operatorname{diam}\left(G_{k}\right)$ would satisfy our requirements. This proves our lemma.

Note that, with the notation from the statement of the previous lemma, one has $a$ fortiori that $B_{r}(p)$ and $B_{r}(q)$ are not isomorphic as colored graphs; in other words, $\alpha_{r}(p) \neq \alpha_{r}(q)$.

Proposition 4.5. The 4-edge-colored graph $G$ is generic.

Proof. Let $x, y$ be two distinct vertices of $G$. By transitivity of the action there exists $\gamma \in \Gamma$ such that $\Phi(\gamma)(x)=p$ (the single vertex of $\left.G_{0}\right)$. Set $q=\Phi(\gamma)(y)$. By the previous lemma, there exists $r$ such that $B_{r}(p)$ is not colored-isomorphic to $B_{r}(q)$. Then $B_{r+|\gamma|}(x)$ is not colored-isomorphic to $B_{r+|\gamma|}(y)$, where $|\gamma|$ is the word-length of $\gamma$.

Proposition 4.6. The 4-edge-colored graph $G$ is of dense holonomy.

Proof. Let $t \in V(G)$ and $r>0$. Let $i=i(t)$ be such that $B_{r}(t) \subset G_{i}$. Put $M=\operatorname{diam}\left(G_{i+3}\right)$.

Lemma 4.7. For any $x \in V(G)$, the ball $B_{M}(x)$ contains a vertex $y$ belonging to a spanned subgraph $L$ isomorphic to $H_{j}$ for some $j \geq i+3$.

Proof. If $x \in V\left(G_{i+2}\right)$ then the statement obviously holds. We proceed by induction. Suppose that the statement holds for any $x \in V\left(G_{\ell}\right)$ with $\ell \geq i+2$. Let $x \in$ $V\left(G_{\ell+1}\right) \backslash V\left(G_{\ell}\right)$. If $x \in H_{\ell+1}$ then $L$ can be chosen as $H_{\ell+1}$ itself. If $x$ is attached to $H_{\ell+1}$ then we have two cases:

- $B_{M}(x)$ intersects $H_{\ell+1}$ : then the statement is clearly true.

- $B_{M}(x)$ does not intersect $H_{\ell+1}$ : then there exists a vertex $x^{\prime} \in V\left(G_{\ell}\right)$ such that $B_{M}(x)$ and $B_{M}\left(x^{\prime}\right)$ are isomorphic, hence the statement follows by induction. 
We continue the proof of the proposition. Let $x \in V(G)$ and let $y \in L \simeq H_{j}$ with $j \geq i+3$, as in the lemma. Then in a (10si+3)-neighborhood of $y$ we attached a copy of $G_{i+3}$. Hence $B_{M+10 s_{i+3}}(y)$ contains a vertex of the same $r$-type as $t$. Therefore, $B_{2 M+10 s_{i+3}}(x)$ contains a vertex of the same $r$-type as the vertex $t$; in other words (recalling the definition of dense holonomy) if $\alpha_{r}(t)=\alpha$, then $m_{\alpha}=2 M+10 s_{i+3}$ (recall that $i=i(t)$ ), and the proposition follows.

\section{Measurable equivalence relations}

5.1. Measurable equivalence relations: definition and examples. Let $(X, \mu)$ be a standard Borel space, where $\mu$ is a probability measure without atoms. A (measurable) equivalence relation on $X$ is a measurable subset $\mathcal{R}$ of $X \times X$ such that the relation $x \sim_{\mathcal{R}} y$ if $(x, y) \in \mathcal{R}$ is an equivalence relation.

Two measurable equivalence relations $\left(X_{1}, \mathcal{R}_{1}\right)$ and $\left(X_{2}, \mathcal{R}_{2}\right)$ are isomorphic if there exists a measurable bijection $\pi: X_{1} \rightarrow X_{2}$ such that

$$
(x, y) \in \mathcal{R}_{1} \text { if and only if }(\pi(x), \pi(y)) \in \mathcal{R}_{2}
$$

for all $x, y \in X_{1}$.

Let now $\Gamma_{0}$ be a countable group and $\alpha$ be a $\Gamma_{0}$-action by $\mu$-preserving measurable automorphisms of $(X, \mu)$. The set $\mathcal{R}_{\alpha} \subset X \times X$ defined by

$$
\mathcal{R}_{\alpha}=\left\{(x, \alpha(\gamma) x) \mid x \in X, \gamma \in \Gamma_{0}\right\}
$$

is the associated orbit equivalence relation on $X$.

Two actions $\alpha_{i}$ of groups $\Gamma_{i}$ on $\left(X_{i}, \mu_{i}\right), i=1,2$, are orbit equivalent if the corresponding orbit equivalence relations $\mathcal{R}_{\alpha_{i}}$ are isomorphic.

5.2. Amenable measurable equivalence relations. Dye [5] showed that any two (non-atomic) probability measure preserving ergodic $\mathbb{Z}$-actions are orbit equivalent.

Subsequently, Dye [6] extended this result to groups of polynomial growth and to infinite abelian groups.

Then Ornstein and Weiss [26] showed that any free ergodic probability measure preserving action of an amenable group is orbit equivalent to an ergodic $\mathbb{Z}$-action. In other words, combining Dye's first result and the latter, one has that all ergodic actions of amenable groups define the same equivalence relation, called the hyperfinite relation (this terminology comes from the fact that there exists an increasing, exhaustive sequence $\left\{\mathcal{R}_{n}\right\}_{n \in \mathbb{N}}$ of finite measurable subequivalence relations of $\left.\mathcal{R}\right)$.

There is a notion of amenability for equivalence relations $(X, \mathcal{R})$ due to R. Zimmer [31]. Hyperfiniteness easily implies amenability; much less obvious, the converse implication was proved by Connes, Feldman and Weiss [2]. See also [19] for another 
approach to these notions which, incidentally, leads to an alternative proof of the Connes-Feldman-Weiss theorem.

Hyperfiniteness can be thought of as a "global"amenability property for equivalence relations. Another property, of a "local" nature, is that a.e. orbits (the Schreier graphs) are Følner. Whereas global amenability always implies local amenability (see, e.g., [2], Lemma 8, and [19], Theorem 1) the converse is, in general, not true. Besides the results of C. Series [29] and M. Samuélidès [28], who proved that foliations of polynomial growth are hyperfinite, Kaimanovich [19], [20] provided several (counter)examples of countable equivalence relations, whose leaves are a.e. Følner, which, however, are not hyperfinite.

Let $\mathcal{R}$ be a measurable-equivalence relation on $(X, \mu)$. Let $S \subset X$ be a measurable subset of positive measure. Denote by $\mu_{S}$ the restriction of $\mu$ to $S$ and by $(\mathcal{R} \mid S)$ the induced equivalence relation on $\left(S, \mu_{S}\right)$. One calls $S$ a complete section for $\mathcal{R}$ if it intersects every class of $\mathcal{R}$.

The following easy result (see, e.g., [21], Proposition 6.9) relates hyperfiniteness of a measurable equivalence relation to the hyperfiniteness of the equivalence relation induced by a complete section.

Proposition 5.1. Let $\mathcal{R}$ be a measurable-equivalence relation on $(X, \mu)$. Suppose that $S \subset X$ is a measurable subset. If $\mathcal{R}$ is hyperfinite, then $(\mathcal{R} \mid S)$ is also hyperfinite. Conversely, if $S$ is a complete section for $\mathcal{R}$ and $(\mathcal{R} \mid S)$ is hyperfinite, then $\mathcal{R}$ is hyperfinite.

5.3. L-graphings. A (locally countable measurable) graph on $(X, \mu)$ is a graph $\mathscr{E}$ with vertex set $X$, the edge set a measurable subset of $X \times X$ and such that every $x \in X$ has at most countably many neighbors. Any such graph defines an equivalence relation $\mathcal{R}_{\mathscr{E}}$ on $X$ : two points $x, y \in X$ satisfy $(x, y) \in \mathcal{R}_{\mathscr{G}}$ if and only if they belong to the same connected component of $\mathscr{E}$, i.e., there exists a finite path in $\mathscr{E}$ connecting them.

If $\mathcal{R}$ is a countable measurable relation, then a (measurable) L-graphing of $\mathcal{R}$ is a graph $\mathcal{E}$ whose connected components are exactly the equivalence classes of $\mathcal{R}$. In other words, $\mathcal{R}_{\mathcal{E}}=\mathcal{R}$, and one says that $\mathscr{E}$ generates $\mathcal{R}$. As an example, if $\alpha$ is an action on $(X, \mu)$ of a finitely generated group $\Gamma_{0}$, say by a finite symmetric generating system $\Sigma$, then the associated Schreier graph $\mathcal{G}\left(\Gamma_{0}, \Sigma, \alpha, X\right)$ (whose vertices are the points $x \in X$ and the edges are the pairs $(x, \alpha(\sigma) x), x \in X$ and $\sigma \in \Sigma)$ generates the associated equivalence relation $\mathcal{R}_{\alpha}$.

5.4. Cost of an equivalence relation. For a group $\Gamma_{0}$ the minimal number of generators is called the rank. Similarly, with an equivalence relation one associates a numerical invariant, called the cost. This concept was introduced by Levitt [23] and developed by Gaboriau [8], [9]. Here is how it is defined. 
Let $\mathcal{R}$ be a measurable equivalence relation on $(X, \mu)$. Then, one defines a measure $M$ on $\mathcal{R}$ by setting, for all measurable $A \subseteq \mathcal{R}$,

$$
M(A)=\int_{X}\left|A_{x}\right| d \mu(x),
$$

where $|\cdot|$ denotes cardinality (possibly infinite) and $A_{x}=\{y \mid(x, y) \in A\}$ for $x \in X$. If $\mathscr{G}$ is a graphing of $\mathcal{R}$, then one defines its cost by

$$
C_{\mu}(\mathscr{E})=\frac{1}{2} M(\mathscr{E})
$$

(the factor $\frac{1}{2}$ is a normalizing constant). Note that in fact one has

$$
C_{\mu}(\mathscr{E})=\frac{1}{2} \int_{X} \operatorname{deg}_{\mathscr{E}}(x) d \mu(x),
$$

where $\operatorname{deg}_{\mathscr{E}}=|\{y \in X \mid(x, y) \in \mathscr{E}\}|$ is the degree of $x$ : for this reason, sometimes one also calls $C_{\mu}(\mathscr{E})$ the edge measure of $\mathscr{E}$.

Finally, the cost $C_{\mu}(\mathcal{R})$ of an equivalence relation $\mathcal{R}$ is the infimum of the costs over all the generating graphings:

$$
C_{\mu}(\mathcal{R})=\inf _{\mathscr{S}: \mathcal{R} g=\mathcal{R}} C_{\mu}(\mathscr{E}) .
$$

This is an invariant of the equivalence relation: orbit equivalent relations have the same cost.

The cost of the hyperfinite ergodic relation is 1 .

An L-treeing is a graphing where graphs associated with almost all $x \in X$ are trees. We have the following remarkable fact ([8]; see also [21], Proposition 19.1 and Theorem 19.1).

Theorem 5.2 (Gaboriau). Let $\mathcal{R}$ be a countable measurable equivalence relation and let $\mu$ be $\mathcal{R}$-invariant with $C_{\mu}(\mathcal{R})<\infty$. Let $\mathcal{E}$ be a graphing of $\mathcal{R}$. Then $C_{\mu}(\mathscr{E})=C_{\mu}(\mathcal{R})$ (i.e., $\mathscr{E}$ realizes the infimum of the costs) if and only if $\mathscr{E}$ is a treeing of $\mathcal{R}$.

In particular, any free action of the free group $\mathbb{F}_{n}$ has cost $n$.

A crucial result of Gaboriau (see [21], Theorem 21.1) relates the cost of an equivalence relation to the cost of the equivalence relation induced by a complete section.

Theorem 5.3 (Gaboriau). $C_{\mu}(\mathcal{R})=C_{\mu \mid S}(\mathcal{R} \mid S)+\mu(X \backslash S)$. 
5.5. The ergodic decomposition theorem. For a countable measurable equivalence relation $\mathcal{R}$ on $X$ we denote by $E_{\mathcal{R}}$ the standard Borel space of ergodic $\mathcal{R}$-invariant probability measures on $X$ (again a measurable subset of the standard Borel space $P(X)$ of probability measures on $X$, see [3]).

The ergodic decomposition theorem of Farrell [7] and Varadarajan [30] (see also [21], Theorem 18.5) states as follows.

Theorem 5.4 (Farrel and Varadarajan). Let $\mathcal{R}$ be a countable measurable equivalence relation on $X$. Assume that there exist $\mathcal{R}$-invariant probability measures on $X$. Then $E_{\mathcal{R}} \neq \emptyset$ and there is a measurable surjection $\pi: X \rightarrow E_{\mathcal{R}}$ such that

(i) $\pi$ is $\mathcal{R}$-invariant;

(ii) if $X_{e}=\{x \mid \pi(x)=e\}, e \in E_{\mathcal{R}}$, then $e\left(X_{e}\right)=1$ (and in fact $e$ is the unique $\mathcal{R}$-ergodic measure on $\left.\mathcal{R}\right|_{X_{e}}$;

(iii) for any $\mathcal{R}$-invariant probability measure $\mu$,

$$
\mu=\int_{X} \pi(x) d \mu(x) \equiv \int_{E_{\mathcal{R}}} e d v_{\mu}(e)
$$

where $v_{\mu}=\pi * \mu \in P\left(E_{\mathcal{R}}\right)$.

Corollary 5.5. With the above notation one has

$$
C_{\mu}(\mathcal{R})=\int_{X} C_{\pi(x)}(\mathcal{R}) d \mu(x)=\int_{E_{\mathcal{R}}} C_{e}(\mathcal{R}) d v_{\mu}(e) .
$$

\section{Measurable equivalence relations on $X_{G}$}

6.1. "Local" amenability. In this section we prove Theorem1.3, namely that for the $\Gamma$-action $\widetilde{\Phi}$ on $X_{G}$ all leaves are Følner. Recall that $\Gamma=\mathbb{Z}_{2} * \mathbb{Z}_{2} * \mathbb{Z}_{2} * \mathbb{Z}_{2}$.

First note that the action $\widetilde{\Phi}$ is regular, that is, if $p \in X_{G}, \gamma \in \Gamma$ then either $\widetilde{\Phi}(\gamma)(p) \neq p$ or the homeomorphism $\widetilde{\Phi}(\gamma)$ fixes a whole neighborhood of $p$. Indeed let $p=\left\{\alpha_{1}, \prec \alpha_{2} \prec \cdots \prec \alpha_{s} \prec \cdots\right\}$ and let the word-length of $\gamma$ be $s$. If $\widetilde{\Phi}(\gamma)(p)=p$, then $\widetilde{\Phi}(\gamma)$ also fixes all $q \in X_{G}$ of the form $q=\left\{\alpha_{1}, \prec \alpha_{2} \prec \cdots \prec\right.$ $\left.\alpha_{s} \prec \beta_{s+1} \prec \cdots\right\}$.

We say that two 4-colored graphs $G_{1}, G_{2}$ are locally isomorphic if $U_{G_{1}}^{r}=U_{G_{2}}^{r}$ for any $r>0$. Clearly if $G_{1}$ is amenable and of dense holonomy and $G_{2}$ is locally isomorphic to $G_{1}$, then $G_{2}$ is amenable and of dense holonomy as well [18]. Since our graph $G$ is generic, the dense leaf $\pi(G) \subset X_{G}$ is isomorphic to $G$. By minimality, regularity and dense holonomy, any two orbits (the corresponding Schreier graphs) are locally isomorphic (see also [15]) and thus our theorem follows. 
As we shall see later, our $\Gamma$-action $\widetilde{\Phi}$ defines a non-hyperfinite measurable equivalence relation with respect to some ergodic invariant measure. As mentioned earlier, the existence of non-hyperfinite relations with Følner leaves was first observed by Kaimanovich [20].

6.2. The invariant measures. Notice that in our main construction we have a parameter $m$ and we have in fact constructed a graph $G^{(m)}$ for each $m>10$. By Lemma 4.1,

$$
V\left(H_{n}^{(m)}\right)=R_{1}^{n,(m)} \amalg R_{2}^{n,(m)} \amalg \cdots \amalg Q^{n,(m)},
$$

where

$$
\frac{\left|R_{i}^{n,(m)}\right|}{\left|V\left(H_{n}^{(m)}\right)\right|}<\frac{1}{m^{i} 2^{\left|V\left(G_{i-1}^{(m)}\right)\right|}}
$$

for $i=1,2, \ldots, n$ so that

$$
\frac{\left|Q^{n,(m)}\right|}{\left|V\left(H_{n}^{(m)}\right)\right|}>1-\frac{1}{m} .
$$

Here the upper index $(m)$ indicates that the parameter is chosen to be $m$. For each vertex of $R_{i}^{n,(m)}$ we attached a copy of a graph of size $\left|V\left(G_{i-1}^{(m)}\right)\right|$. We also attached a path of length at most $n$ to one vertex of $Q^{n,(m)}$. From (4.4) we have the following inequality:

$$
\frac{\left|V\left(G_{n}^{(m)}\right) \backslash V\left(H_{n}^{(m)}\right)\right|}{\left|V\left(H_{n}^{(m)}\right)\right|}<\frac{1}{m} .
$$

Let $F_{n}^{(m)}:=V\left(G_{n}^{(m)}\right) \backslash V\left(G_{n-1}^{(m)}\right)$. Clearly $\left|\partial F_{n}^{(m)}\right|=2$, thus $\left\{F_{n}^{(m)}\right\}_{n=1}^{\infty}$ forms a Følner sequence in $G^{(m)}$. Note that such a sequence is exhausting (i.e., $\bigcup_{n=0}^{\infty} F_{n}^{(m)}=$ $\left.V\left(G_{n}^{(m)}\right)\right)$, though not increasing.

We now consider two new Følner sequences for $G^{(m)}:\left\{F_{n}^{1,(m)}\right\}_{n=1}^{\infty}$ and $\left\{F_{n}^{2,(m)}\right\}_{n=1}^{\infty}$, where $F_{n}^{1,(m)}=F_{2 n}^{(m)}$ and $F_{n}^{2,(m)}=F_{2 n+1}^{(m)}$. By the averaging process described in Proposition3.2 we then obtain two invariant measures, $\mu_{1}^{(m)}$ and $\mu_{2}^{(m)}$, on $X_{G^{(m)}}$.

Thus, for each $m>10$ we have

- a compact metric space $X_{G^{(m)}}$;

- a minimal $\Gamma$-action $\widetilde{\Phi}^{(m)}$;

- an L-graphing $\mathscr{E}^{(m)}$ associated with the action;

- a measurable-equivalence relation $\mathcal{R}^{(m)}$ defined by $\mathscr{E}^{(m)}$;

- two $\mathcal{R}^{(m)}$-invariant measures, $\mu_{1}^{(m)}$ and $\mu_{2}^{(m)}$. 
6.3. Lower estimate for the cost. A point $x \in X_{G^{(m)}}$ is called $\widetilde{\Gamma}$-free if $\widetilde{\Phi}(\gamma)(x) \neq x$ for any $\gamma \in \widetilde{\Gamma} \backslash\{1\}$. Clearly the set $S^{(m)} \subset X_{G^{(m)}}$ of $\widetilde{\Gamma}$-free points is closed.

Lemma 6.1. $\lim _{m \rightarrow \infty} \mu_{2}^{(m)}\left(S^{(m)}\right)=1$.

Proof. Let $S_{k}^{(m)}$ be the set of points in $X_{G^{(m)}}$ for which $\widetilde{\Phi}(\gamma)(x) \neq x$ whenever the word-length of $\gamma \in \widetilde{\Gamma}$ is not greater than $k$. Clearly, $\bigcap_{k=1}^{\infty} S_{k}^{(m)}=S^{(m)}$. Observe that

$$
\mu_{2}^{(m)}\left(S_{k}^{(m)}\right)=\lim _{\omega} \frac{\left|W_{n, k}^{(m)}\right|}{\left|F_{n}^{2,(m)}\right|},
$$

where $W_{n, k}^{(m)}$ is the set of vertices $p$ in $F_{n}^{2,(m)}$ such that $\Phi(\gamma)(p) \neq p$, if $|\gamma| \leq k$. Obviously, if $p \in H_{n}^{(m)}$ and $n$ is a large odd number, then $p \in W_{n, k}^{(m)}$. Hence $\mu_{2}^{(m)}\left(S_{k}^{(m)}\right) \geq 1-\frac{2}{m}$. Therefore $\mu_{2}^{(m)}\left(S^{(m)}\right) \geq 1-\frac{2}{m}$ as well.

Lemma 6.2. The induced equivalence relation $\left(\mathcal{R}^{(m)} \mid S^{(m)}\right)$ on the set of $\tilde{\Gamma}$-free points is exactly the relation given by the $\widetilde{\Gamma}$-action.

Proof. Notice that in the graph $G^{(m)}$ there is no simple cycle containing an edge colored by $D$. Hence the same holds for the orbits in $X_{G^{(m)}}$. Thus if $p, q \in S^{(m)}$ and $\gamma$ contains the symbol $D$, then $\widetilde{\Phi}(\gamma)(p) \neq q$.

Lemma 6.3. $C_{\mu_{2}^{(m)}}\left(\mathcal{R}^{(m)} \mid S^{(m)}\right)=\frac{3}{2} \mu_{2}^{(m)}\left(S^{(m)}\right)$.

Proof. This is a simple consequence of Theorem5.2 which can be rephrased as follows: the cost of an equivalence relation defined by an $L$-treeing $\mathscr{G}$ is exactly the edge measure of $\mathscr{G}$.

It then suffices to note that the edge measure of a free $\mathbb{Z}_{2} * \mathbb{Z}_{2} * \mathbb{Z}_{2}$-action on a compact metric space with an invariant measure is $\frac{3}{2}$ times the measure of the space.

Lemma 6.4. $S^{(m)}$ is a complete section of $\mathcal{R}^{(m)}$.

Proof. We need to prove that the set of points $p \in X_{G^{(m)}}$, such that there exists no $\gamma \in \Gamma$ with $\widetilde{\Phi}(\gamma)(p) \in S^{(m)}$ has $\mu_{2}^{(m)}$-measure zero. We denote this set by $B^{(m)}$.

Let $S_{k, n} \subset F_{n}^{2,(m)}$ be the set of vertices $p$ such that $d_{G_{n}}\left(p, H_{2 n+1}^{(m)}\right) \leq k$. By our construction of $G^{(m)}$, it is clear that for any $\varepsilon>0$ there exists $k_{\varepsilon}$ such that

$$
\frac{\left|S_{k_{\varepsilon}, n}\right|}{\left|F_{n}^{2,(m)}\right|} \geq 1-\varepsilon
$$


Therefore $\mu_{2}^{(m)}\left(\Theta_{k_{\varepsilon}}\right) \geq 1-\varepsilon$, where $\Theta_{k_{\varepsilon}}$ is the set of points $x \in X_{G^{(m)}}$ for which there exists $\delta \in \Gamma$ with $|\delta| \leq k_{\varepsilon}$ such that $\widetilde{\Phi}(\delta)(x) \in S^{(m)}$. Thus $\mu_{2}^{(m)}\left(B^{(m)}\right)=0$.

The following proposition is the straightforward corollary of the above lemmas and of Theorem 2.2.

Proposition 6.5. $\lim _{m \rightarrow \infty} C_{\mu_{2}^{(m)}}\left(\mathcal{R}^{(m)}\right)=\frac{3}{2}$.

6.4. The hyperfinite relation. Let $D_{\infty}=\langle A, B\rangle \cong \mathbb{Z}_{2} * \mathbb{Z}_{2}$ denote the subgroup of $\Gamma$ generated by the two involutions $A$ and $B$. This is the infinite dihedral group. In analogy with the $\widetilde{\Gamma}$-case, we have that the set $S_{(m)} \subset X_{G^{(m)}}$ of $D_{\infty}$-free points is a complete section of $\mathcal{R}^{(m)}$ (with respect to the measure $\mu_{1}^{(m)}$ which is "concentrated" on the cycles $C_{i}$ 's). Since $D_{\infty}$ is amenable, we have that the induced equivalence relation $\left(\mathcal{R}^{(m)} \mid S_{(m)}\right)$ is hyperfinite. From Proposition 5.1, we then deduce the following.

Proposition 6.6. The measurable equivalence relation $\left(\widetilde{\Phi}^{(m)}, X_{G^{(m)}}, \mu_{1}^{(m)}\right)$ is hyperfinite for all $m>10$.

With a little more work we can, in fact, also show that $\mu_{1}^{(m)}$ can be chosen to be ergodic. Let $\left\{\mathcal{R}_{n}^{(m)}\right\}_{n \in \mathbb{N}}$ be a sequence of finite subequivalence relations converging to $\mathcal{R}^{(m)}$. Denote by $\mathscr{E}_{n}^{(m)}$ and $\mathscr{E}^{(m)}$ the corresponding graphings.

Let $f(n)=C_{\mu_{1}^{(m)}}\left(\mathscr{E}^{(m)} \backslash \mathscr{E}_{n}^{(m)}\right)$ denote the edge-measure of the edges in $\mathscr{G}^{(m)}$ which are not in $\mathscr{E}_{n}^{(m)}$. Then $f(n)$ monotonically converges to zero. By the ergodic decomposition theorem we have

$$
f(n)=\int_{E_{\mathcal{R}}} C_{\boldsymbol{e}}\left(\mathscr{E}^{(m)} \backslash \mathscr{E}_{n}^{(m)}\right) d \nu_{\mu_{1}^{(m)}}(e) .
$$

Beppo Levi's Theorem then implies that, for almost all $e \in E_{\mathcal{R}}$, the sequence $\left\{C_{e}\left(\mathscr{G}^{(m)} \backslash \mathscr{E}_{n}^{(m)}\right)\right\}_{n \in \mathbb{N}}$ also converges to zero, showing that the corresponding equivalence relation is hyperfinite.

In other words, the ergodic decomposition of a hyperfinite measure is the direct integral of hyperfinite ergodic measures.

6.5. The proof of the main result. We can now prove our main result (Theorem1.2).

In our setting we have $\Gamma=\mathbb{Z}_{2} * \mathbb{Z}_{2} * \mathbb{Z}_{2} * \mathbb{Z}_{2}$ and $C=X_{G}$, where $X_{G}$ is the type space of the 4-regular, 4-properly edge-colored graph $G$ of our main construction, and $\widetilde{\Phi}$ is the associated minimal topological action of $\Gamma$.

We want to show that there exist two ergodic invariant measures $\mu_{1}$ and $\mu_{2}$ on $X_{G}$ such that the corresponding measurable equivalence relations $\left(\widetilde{\Phi}, X_{G}, \mu_{1}\right)$ and $\left(\widetilde{\Phi}, X_{G}, \mu_{2}\right)$ are not orbit equivalent and, in addition, the first one is hyperfinite. 
By Proposition 6.5, there exists large enough $\bar{m} \in \mathbb{N}$ such that the cost of the equivalence relation associated with $\left(\widetilde{\Phi}^{(\bar{m})}, X_{G^{(\bar{m})}}, \mu_{2}^{(\bar{m})}\right)$ is $\geq 5 / 4$. Set $G=G^{(\bar{m})}$, $\Phi=\Phi^{(\bar{m})}, \mu_{1}=\mu_{1}^{(\bar{m})}$ and $\mu_{2}^{\prime}=\mu_{2}^{(\bar{m})}$. By the preceding section, we can suppose that $\mu_{1}$ is ergodic (hyperfinite).

On the other hand, by the ergodic decomposition theorem, since $5 / 4 \leq C_{\mu_{2}^{\prime}}(\mathcal{R})$, there exists an ergodic invariant measure $\mu_{2}$ with $C_{\mu_{2}}(\mathcal{R}) \neq 1$. Thus $\left(\widetilde{\Phi}, X_{G}, \mu_{2}\right)$ is not hyperfinite, which completes the proof.

\section{A remark on the type space}

Let $G=(V, E)$ be a graph and $x \in V$ be a specified vertex. The pair $(G, x)$ is called a marked graph based at $x$. Given an integer $k \geq 2$, we denote by $X_{k}$ the space of all marked, $k$-regular, properly $k$-edge colored graphs (possibly with multiple loops). Then $X_{k}$ becomes a metric space by introducing a distance function $d: X_{k} \times X_{k} \rightarrow$ $[0,1]$ defined by setting $d\left((G, x)\left(G^{\prime}, x^{\prime}\right)\right)=2^{-r}$ for all $(G, x),\left(G^{\prime}, x^{\prime}\right) \in X_{k}$, where $r=\sup \left\{n \geq 0 \mid B_{G}(n, x) \cong B_{G^{\prime}}\left(n, x^{\prime}\right)\right.$ as labeled graphs\}. It is easy to see that $X_{k}$ is compact (see [17], Theorem 3 ).

In a group theoretical framework, this notion was developed in the following sense. Let $F_{m}$ be the free group of rank $m \geq 2$. Consider the set $\mathcal{P}\left(F_{m}\right)$ of all subsets of $F_{m}$ and its natural direct product (metrizable) topology (this is the topology where the basis consists of open cylindrical sets $U_{A, B}=\left\{N \in \mathcal{P}\left(F_{n}\right) \mid A \subset N, B \cap N=\emptyset\right\}$, with $A$ and $B$ arbitrary finite subsets of $F_{m}$ ). This topology was introduced by Grigorchuk in [13] and is often called the Cayley-Grigorchuk topology (the full terminology will become clear in the next paragraph); see also [1] and the survey [14]. We also mention the recent paper [25], where it is constructed an isomorphism-invariant subset of $\mathscr{E}_{3}$ homeomorphic to the Cantor set such that the isomorphism classes are dense in it (minimality). Also recall that for locally compact groups an analogous notion exists and the corresponding topology is called the Chabauty topology.

Denote by $\mathscr{E}_{k}$ the subspace of $X_{k}$, consisting of all (marked) Cayley graphs of $k$-generated groups (with symmetric generating systems!), equipped with the induced topology from $X_{k}$. Then the map $\mathscr{E}_{2 m} \ni \Gamma \rightarrow N(\Gamma) \in \mathcal{P}\left(F_{m}\right)$, where $N(\Gamma)$ is the kernel of the canonical quotient homomorphism $F_{m} \rightarrow \Gamma$, is an isometry.

Let now $G$ be a $k$-regular properly $k$-edge colored graph with dense holonomy and denote by $X_{G}$ the corresponding type space (cf. Section3). Introduce the distance $d^{\prime}: V(G) \times V(G) \rightarrow[0,1]$ defined by $d^{\prime}(x, y)=2^{-r}$. Here $r=\sup \{n \geq 0 \mid$ $\left.\alpha_{r}(x)=\alpha_{r}(y)\right\}$ for all $x, y \in V(G)$. Then, tautologically, the map $\Psi: V(G) \rightarrow X_{k}$, defined by setting $\Psi(x)=(G, x)$ for all $x \in V(G)$, is an isometry and therefore extends to a map $\widetilde{\Psi}: X_{G} \rightarrow X_{k}$. Indeed,

$$
\widetilde{\Psi}\left(\left\{\alpha_{1} \preceq \alpha_{2} \preceq \cdots\right\}\right)=\lim _{r \rightarrow \infty}\left(G, x_{r}\right),
$$


where $x_{r} \in V(G)$ is such that $\alpha\left(x_{r}\right)=\alpha_{r}$ for all $r=1,2, \ldots$. Note that the limit on the right-hand side exists and is a well-defined marked, $k$-regular, properly $k$-edge colored graph (cf. [18], Section 6.A). It follows that $X_{G}$ is homeomorphic to the closure $\overline{\{(G, x) \mid x \in V(G)\}} \subset X_{k}$. For such limits of (marked) Schreier graphs see [16], Theorem $18.5 \mathrm{~N}$, where very interesting fractal sets are constructed from Schreier graphs of so-called self-similar groups [24]. Note that similar limit objects, called Benjamini-Schramm limits, are studied in the theory of graph percolation.

Acknowledgments. We express our deepest gratitude to the referee for her/his most careful reading of our manuscript and for the several remarks and suggestions that undoubtedly improved the presentation of our paper. We also thank Rostislav I. Grigorchuk and Volodia Nekrashevych for their interest and for stimulating discussions (leading, in particular, to the final remark).

G. E. was supported by OTKA Grants T 049841 and T 037846, and, at the initial stage of the present research, by GNAMPA-INdAM.

\section{References}

[1] C. Champetier, L'espace des groupes de type fini. Topology 39 (2000), 657-680. Zbl 0959.20041 MR 1760424

[2] A. Connes, J. Feldman, and B. Weiss, An amenable equivalence relation is generated by a single transformation. Ergodic Theory Dynam. Systems 1 (1981), 431-450 (1982). Zbl 0491.28018 MR 662736

[3] R. Dougherty, S. Jackson, and A. S. Kechris, The structure of hyperfinite Borel equivalence relations. Trans. Amer. Math. Soc. 341 (1994), 193-225. Zbl 0803.28009 MR 1149121

[4] E. K. van Douwen, Measures invariant under actions of $F_{2}$. Topology Appl. 34 (1990), 53-68. Zbl 0701.43001 MR 1035460

[5] H. A. Dye, On groups of measure preserving transformation. I. Amer. J. Math. 81 (1959), 119-159. Zbl 0087.11501 MR 0131516

[6] H. A. Dye, On groups of measure preserving transformations. II. Amer. J. Math. 85 (1963), 551-576. Zbl 0191.42803 MR 0158048

[7] R. H. Farrell, Representations of invariant measures. Illinois J. Math. 6 (1962), 447-467. Zbl 0108.14203 MR 0150264

[8] D. Gaboriau, Mercuriale de groupes et de relations. C. R. Acad. Sci. Paris Sér. I Math. 326 (1998), 219-222. Zbl 1007.28012 MR 1646912

[9] D. Gaboriau, Coût des relations d'équivalence et des groupes. Invent. Math. 139 (2000), 41-98. Zbl 0939.28012 MR 1728876

[10] D. Gaboriau, On orbit equivalence of measure preserving actions. In Rigidity in dynamics and geometry (Cambridge, 2000), Springer-Verlag, Berlin 2002, 167-186. Zbl 1036.22008 MR 1919400 
[11] Y. Glasner and N. Monod, Amenable actions, free products and a fixed point property. Bull. Lond. Math. Soc. 39 (2007), 138-150. Zbl 05139551 MR 2303529

[12] F. P. Greenleaf, Invariant means on topological groups and their applications. Van Nostrand Math. Stud. 16, Van Nostrand Reinhold Co., New York 1969. Zbl 0174.19001 MR 0251549

[13] R. I. Grigorchuk, Degrees of growth of finitely generated groups and the theory of invariant means. Izv. Akad. Nauk SSSR Ser. Mat. 48 (1984), 939-985; English transl. Math. USSRIzv. 25 (1985) , 259-300. Zbl 0583.20023 MR 764305

[14] R. I. Grigorchuk, Solved and unsolved problems around one group. In Infinite groups: geometric, combinatorial and dynamical aspects, Progr. Math. 248, Birkhäuser, Basel 2005, 117-218. Zbl 05152179 MR 2195454

[15] R. Grigorchuk and V. Nekrashevych, Amenable actions of nonamenable groups. Zap. Nauchn. Sem. S.-Peterburg. Otdel. Mat. Inst. Steklov. (POMI) 326 (2005), 85-96; English transl. J. Math. Sci. 140 (2007), 391-397. Zbl 1127.43001 MR 2183217

[16] R. I. Grigorchuk, V. V. Nekrashevych and V. I. Sushchanskii, Automata, dynamical systems, and groups. Trudy Mat. Inst. Steklov. 231 (2000), 134-214; English transl. Proc. Steklov Inst. Math. 231 (2000), 128-203. Zbl 01729301 MR 1841755

[17] R. I. Grigorchuk and A. Żuk, On the asymptotic spectrum of random walks on infinite families of graphs. In Random walks and discrete potential theory (Cortona, 1997), Sympos. Math. XXXIX, Cambridge University Press, Cambridge 1999, 188-204. Zbl 0957.60044 MR 1802431

[18] M. Gromov, Endomorphisms of symbolic algebraic varieties. J. Eur. Math. Soc. 1 (1999), 109-197. Zbl 0998.14001 MR 1694588

[19] V. A. Kaimanovich, Amenability, hyperfiniteness, and isoperimetric inequalities. $C$. $R$. Acad. Sci. Paris Sér. I Math. 325 (1997), 999-1004. Zbl 0981.28014 MR 1485618

[20] V. A. Kaimanovich, Equivalence relations with amenable leaves need not be amenable. In Topology, ergodic theory, real algebraic geometry, Amer. Math. Soc. Transl. Ser. (2) 202, Amer. Math. Soc., Providence, RI 2001, 151-166. Zbl 0990.28013 MR 1819187

[21] A. S. Kechris and B. D. Miller, Topics in orbit equivalence. Lecture Notes in Math. 1852, Springer-Verlag, Berlin 2004. Zbl 1058.37003 MR 2095154

[22] J. C. Lagarias and P. A. B. Pleasants, Local complexity of Delone sets and crystallinity. Canad. Math. Bull. 45 (2002), 634-652, Zbl 1016.52013 MR 1941231

[23] G. Levitt, On the cost of generating an equivalence relation. Ergodic Theory Dynam. Systems 15 (1995), 1173-1181. Zbl 0843.28010 MR 1366313

[24] V. Nekrashevych, Self-similar groups. Math. Surveys Monogr. 117, Amer. Math. Soc., Providence, RI, 2005. Zbl 1087.20032 MR 2162164

[25] V. Nekrashevych, A minimal Cantor set in the space of 3-generated groups. Geom. Dedicata 124 (2007), 153-190. Zbl 05180055 MR 2318543

[26] D. S. Ornstein and B. Weiss, Ergodic theory of amenable group actions. I: The Rohlin lemma. Bull. Amer. Math. Soc. (N.S.) 2 (1980), 161-164. Zbl 0427.28018 MR 551753 
[27] J. C. Oxtoby, Ergodic sets. Bull. Amer. Math. Soc. 58 (1952), 116-136. Zbl 0046.11504 MR 0047262

[28] M. Samuelides, Tout feuilletage à croissance polynomiale est hyperfini. J. Funct. Anal. 34 (1979), 363-369. Zbl 0491.28019 MR 556261

[29] C. Series, Foliations of polynomial growth are hyperfinite. Israel J. Math. 34 (1979), 245-258 (1980). Zbl 0436.28015 MR 570884

[30] V. S. Varadarajan, Groups of automorphisms of Borel spaces. Trans. Amer. Math. Soc. 109 (1963), 191-220. Zbl 0192.14203 MR 0159923

[31] R. J. Zimmer, Amenable ergodic actions, hyperfinite factors, and Poincaré flows. Bull. Amer. Math. Soc. 83 (1977), 1078-1080. Zbl 0378.28008 MR 0460598

Received November 7, 2006; revised January 7, 2008

T. Ceccherini-Silberstein, Dipartimento di Ingegneria, Università del Sannio, Corso Garibaldi 107, 82100 Benevento, Italy

E-mail: tceccher@mat.uniroma1.it

G. Elek, Alfred Renyi Mathematical Institute of the Hungarian Academy of Sciences, P.O. Box 127, 1364 Budapest, Hungary

E-mail: elek@renyi.hu 